\title{
Dynamic Conductivity of Electrons and Electron-Phonon Interaction in Open Three-Well Nanostructures
}

\author{
M.V. TKach*, Ju.O. Seti, Y.B. GRynyshyn and O.M. VoitsekhivskA \\ Chernivtsi National University, Kotsiybynsky str. 2, 58012, Chernivtsi, Ukraine
}

(Received February 24, 2015; in final form June 5, 2015)

\begin{abstract}
The theoretical description of electronic tunneling transport through the three-well nanostructure $\left(\mathrm{In}_{0.53} \mathrm{Ga}_{0.47} \mathrm{As} / \mathrm{In}_{0.52} \mathrm{Al}_{0.48} \mathrm{As}\right)$, being an expanded active region of quantum cascade detector, is presented. Using the solution of the Schrödinger equation, the dynamic conductivity caused by quantum transitions due to the interaction of electrons with electromagnetic field and phonons is calculated. Within the Green functions approach, the electron spectrum, renormalized due to the interaction with confined optical and interface phonons is obtained at cryogenic and room temperatures. The role of different mechanisms of electron-phonon interaction in the formation of temperature shifts, decay rates of electron states and electromagnetic field absorption bands is investigated. It is shown that independently of the temperature, the contribution produced by interface phonons into renormalized electron spectrum is several times bigger than that of confined phonons. However, the experimentally observed long-wave shift and broadening of absorption band at higher temperatures is, mainly, caused by the decreasing heights of resonant tunneling structure potential barriers.
\end{abstract}

DOI: 10.12693/APhysPolA.128.343

PACS: 63.20Kd, 78.67.De, 72.10.Di

\section{Introduction}

The experimental producing of first quantum cascade lasers (QCL) [1,2] and quantum cascade detectors (QCD) $[3,4]$ stimulates the intensive experimental [5$10]$ and theoretical [11-16] investigation of physical processes in these nanodevices. Such multi-layer nanostructures have the characteristic potential profile for the electrons, which tunnel from the input potential well into the output one, performing quantum transitions between the quasi-stationary states, accompanied by emitting or absorption of electromagnetic field quantum.

At first, the experiments, particularly concerning QCL $[1,2]$, were held at cryogenic temperatures. In theoretical papers [11-16], the spectra and quantum transitions were studied for the closed resonant tunneling structure (RTS) placed into the outer medium-barrier. The transport properties of electronic currents through the open multi-layer RTS placed into the outer mediumwell were studied in theoretical papers [17-20]. The effect of phonons was assumed as not essential, thus the main attention was paid to the spectral and transport characteristics depending on physical parameters and geometrical design of RTS.

Further, the temperature range of functioning devices, in particular QCD [3], enlarged till the room temperatures [5-10]. This fact, together with the other reasons was expected taking into account the phonon subsystem for the theoretical models describing the electron-field interaction arising during the electrons transport through the multi-layer RTS.

* corresponding author; e-mail: ktf@chnu.edu.ua
In the first experimental paper [3], it was shown that QCD fabricated of $\operatorname{In}_{0.53} \mathrm{Ga}_{0.47} \mathrm{As} / \mathrm{In}_{0.52} \mathrm{Al}_{0.48} \mathrm{As}$ cascades successfully operated both at cryogenic $(T=10 \mathrm{~K})$ and room $(T=300 \mathrm{~K})$ temperatures. Analysis of the results proved that rather wide electromagnetic field absorption band $(\Gamma \approx 10 \mathrm{meV})$ with maximal responsivity at wavelength $(\lambda=5.35 \mu \mathrm{m})$ and $T=10 \mathrm{~K}$, became wider and shifted into the long wave region when the temperature increased till the room one.

In the theoretical models describing the closed RTS [11-16] developed without taking into account the interaction between electrons and dissipative systems, the stationary states (between which the quantum transitions occur due to electron-field interaction) are characterized by energies (without decay rates) and oscillator forces of these transitions. At the same conditions, in the open models of RTS [17-22], the quasi-stationary states with finite life times arise, thus, the tunneling transport of electrons is observed. The electronic dynamic conductivity and rather wide electromagnetic field absorption band is observed here even without taking into account the dissipative subsystems.

As far as the phonon occupation numbers are sensitive to the temperature, one can expect that electron-phonon interaction should affect on the temperature dependence of QCD operating parameters. Now then, the investigation of this interaction in multi-layer RTS is important not only to display its negative role in the renormalized electron spectrum and, thus, in the variation of absorption band parameters but, also, because according to the idea of paper [3], the phonons play the key positive role in the functioning of QCD. Just the electron-phonon interaction in extractor "phonon ladder" causes the relaxation of electrons from the excited quasi-stationary state of active region of previous cascade into the ground quasistationary state of the nest one. 
The theory of electron-phonon interaction in one- and two-well closed nanostructures was developed in many papers ([13, 23-29] with references) using the model of effective mass for the electrons and dielectric continuum model for the confined optical (L) and interface (I) phonons. For these models, the electron-phonon Hamiltonian was written in the representation of phonon occupation numbers and in coordinate one over the electron variables. The spectrum of I-phonons in multilayer nanostructures, as a rule, was obtained within the transfer matrix approach. The probabilities of quantum transitions between electron states were found using the Fermi golden rule at different temperatures and design of RTS. As far as we know, in the cited and other papers, the theory of electron-phonon interaction does not explain the small broadening and long wave shift of electromagnetic field absorption band observed during the experiment [3] at the increasing temperature of QCD.

In this paper we present the results of theoretical description, compared to the experimental data, of temperature dependences of electronic dynamic conductivity and electromagnetic field absorption band for the threewell RTS being an expanded active region of QCD, taking into account the varying heights of potential barriers and electron-phonon interaction.

\section{Transmission coefficient and dynamic conductivity of electrons in the open three-well RTS}

The plane open (o) three-well RTS with geometrical parameters shown in Fig. 1 is studied in Cartesian coordinate system with $O Z$ axis perpendicular to the planes of nanostructure. The electron Hamiltonian has the form

$$
H_{\mathrm{e}}^{\mathrm{o}}(\boldsymbol{r})=T_{\rho}+H_{\mathrm{e}}^{(\mathrm{o})}(z) . \quad\left(r=\rho+n_{z} z\right) .
$$

Here $T_{\rho}$ is the kinetic energy of electron, moving in the direction parallel to the planes of RTS,

$$
H_{\mathrm{e}}^{(\mathrm{o})}(z)=-\frac{\hbar^{2}}{2} \frac{\partial}{\partial z} \frac{1}{m(z)} \frac{\partial}{\partial z}+U_{\mathrm{e}}^{(\mathrm{o})}(z)
$$

is the term describing the kinetic energy in the direction perpendicular to the planes of RTS with electron effective mass $m(z)$ in the field $U_{\mathrm{e}}^{(\mathrm{o})}(z)$ of the rectangular potential profile of open system

$$
m(z)=\left\{\begin{array}{l}
m_{\mathrm{w}} ; \\
m_{\mathrm{b}} ;
\end{array} \quad U_{\mathrm{e}}^{(\mathrm{o})}(z)=\left\{\begin{array}{l}
0, \text { wells }, \\
U, \text { barriers. }
\end{array}\right.\right.
$$

The mono-energetic current of uncoupling electrons with concentration $\left(n_{0}\right)$, moving perpendicularly to the planes of RTS, interacts with electromagnetic field characterized by frequency $(\omega)$ and intensity $(F)$. In dipole approximation, the Hamiltonian of electron-field interaction in RTS has the form

$$
H(z, t)=-2 e F z\left[\theta\left(z-z_{-1}\right)-\theta\left(z-z_{7}\right)\right] \cos \omega t .
$$

Assuming, for the sake of simplicity, and according to the experiment that the kinetic energy of electron moving along the RTS is much bigger than its energy in transversal direction $\left(T_{\rho}\right)$, we obtain the complete one- dimensional Schrödinger equation

$$
\begin{aligned}
& \mathrm{i} \hbar \frac{\partial \Psi^{(\mathrm{o})}(E, \omega, z, t)}{\partial t}= \\
& \quad\left[H_{\mathrm{e}}^{(\mathrm{o})}(z)+H(z, t)\right] \Psi^{(\mathrm{o})}(E, \omega, z, t) .
\end{aligned}
$$

It has the exact solutions in each part of RTS $(j=$ $0,1, \ldots, 8)$, thus, the wave function of electron-photon system which takes into account all field harmonics is written as

$$
\begin{aligned}
& \Psi^{(\mathrm{o})}(E, \omega, z, t)= \\
& \sum_{j=0}^{8} \Psi_{j}^{(\mathrm{o})}(E, \omega, z, t)\left[\theta\left(z-z_{j-1}\right)-\theta\left(z-z_{j}\right)\right] .
\end{aligned}
$$

Here

$$
\begin{aligned}
& \Psi_{j}^{(\mathrm{o})}(E, \omega, z, t)=\sum_{p=-\infty}^{\infty} \mathrm{e}^{-\frac{\mathrm{i}}{\hbar}(E+p \hbar \omega) t} \sum_{\ell= \pm} A_{p j}^{(\ell)} \mathrm{e}^{\mathrm{i} \ell K_{p j} z} \\
& \quad \times\left\{\begin{array}{c}
1, \quad p=0,8 \\
\mathrm{e}^{\mathrm{i}\left[\alpha(z) \sin \omega t+\ell \beta_{p j} \cos \omega t\right]}, \quad p=1-7,
\end{array}\right.
\end{aligned}
$$

where

$$
\begin{aligned}
& \alpha(z)=\frac{2 e F z}{\hbar \omega}, \quad \beta_{p j}=\frac{2 e F K_{p j}}{m_{j} \omega^{2}}, \\
& K_{p j}=\left\{\begin{array}{cc}
k_{p}=\hbar^{-1} \sqrt{2 m_{\mathrm{w}}(E+p \hbar \omega)}, & j=0,2,4,6,8, \\
\chi_{p}=\hbar^{-1} \sqrt{2 m_{\mathrm{b}}(E+p \hbar \omega-U)}, & j=1,3,5,7 .
\end{array}\right.
\end{aligned}
$$

$E$ is the electron energy, $\alpha, \beta_{p j}$ are the time-independent coefficients, $z_{-1} \rightarrow-\infty, z_{6} \rightarrow+\infty$.

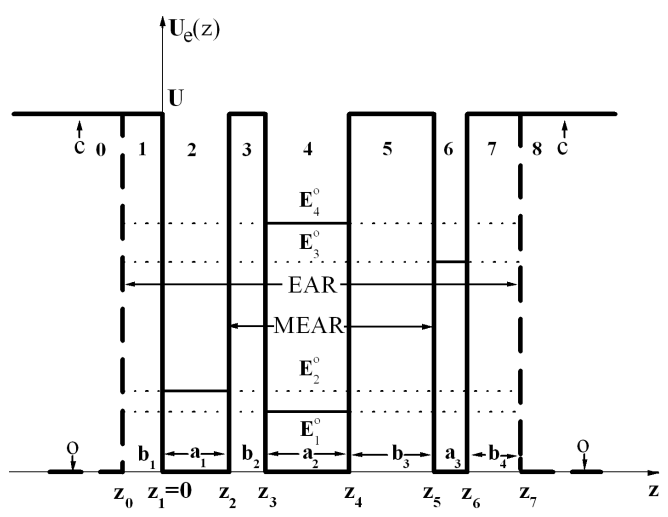

Fig. 1. Scheme of potential profiles and electron energies in open (o) and closed (c) three-well resonant tunneling structure.

The complicated wave functions (7) are essentially simplified for the actual case when the three-well RTS is an active element of experimental QCDs [5-10], detecting the weak electromagnetic fields satisfying the condition $e F d \ll U$, where $d$ is the size of nanostructure.

Expanding the periodical functions $\Psi_{j}^{(\mathrm{o})}$, expression (7), into the Fourier range over the Bessel functions and preserving the linear term over the field intensity $(F)$, we obtain the wave functions of electron-photon system in the so-called small signal one-mode $(p=0, \pm 1)$ 
approximation

$$
\begin{gathered}
\tilde{\Psi}_{j}^{(\mathrm{o})}(E, \omega, z, t)=\mathrm{e}^{-\mathrm{i} E t / \hbar} \sum_{\ell= \pm} \\
\times\left\{\begin{array}{l}
A_{0 j}^{(\ell)} \mathrm{e}^{\mathrm{i} \ell k_{0} z}+A_{1 j}^{(\ell)} \mathrm{e}^{\mathrm{i} \ell k_{1} z} \mathrm{e}^{-\mathrm{i} \omega t} \\
+A_{-1 j}^{(\ell)} \mathrm{e}^{\mathrm{i} \ell k_{-1} z} \mathrm{e}^{\mathrm{i} \omega t}, \\
A_{0 j}^{(\ell)} \mathrm{e}^{\mathrm{i} \ell K_{0 j} z}\left[1+\frac{1}{2}\left(\mathrm{i} \ell \beta_{0 j}+\alpha\right) \mathrm{e}^{\mathrm{i} \omega t}\right. \\
\left.+\frac{1}{2}\left(\mathrm{i} \ell \beta_{0 j}-\alpha\right) \mathrm{e}^{-\mathrm{i} \omega t}\right]+A_{1 j}^{(\ell)} \mathrm{e}^{\mathrm{i} \ell K_{1 j} z} \\
{\left[\mathrm{e}^{-\mathrm{i} \omega t}+\frac{1}{2}\left(\mathrm{i} \ell \beta_{1 j}+\alpha\right)\right]} \\
+A_{-1 j}^{(\ell)} \mathrm{e}^{\mathrm{i} \ell K_{-1 j} z}\left[\mathrm{e}^{\mathrm{i} \omega t}+\frac{1}{2}\left(\mathrm{i} \ell \beta_{-1 j}-\alpha\right)\right], j=1 \div 7
\end{array}\right.
\end{gathered}
$$

The electronic current with energy $(E)$ impinges at RTS from the left hand side, since $A_{ \pm 1,0}^{(+)}=0$ and $A_{p 8}^{(-)}=0,(p=0, \pm 1)$. The other unknown coefficients $\left(A_{p j}^{( \pm)}\right)$are fixed by the conditions of continuity of each harmonic of complete wave function and their densities of currents at all RTS interfaces in any moment of time $(t)$ :

$$
\left.\begin{array}{l}
\Psi_{j}^{(\mathrm{o})}\left(E, \omega, z_{j}, t\right)=\Psi_{j+1}^{(\mathrm{o})}\left(E, \omega, z_{j}, t\right) \\
\left.\frac{1}{m_{j}} \frac{\partial}{\partial z} \Psi_{j}^{(\mathrm{o})}(E, \omega, z, t)\right|_{z=z_{j}}= \\
\left.\frac{1}{m_{j+1}} \frac{\partial}{\partial z} \Psi_{j+1}^{(\mathrm{o})}(E, \omega, z, t)\right|_{z=z_{j}}
\end{array}\right\}(j=0 \div 7) .
$$

The coefficients $\left(A_{p j}^{(\ell)}\right)$ obtained from this system of equations are the linear functions of field intensity $(F)$ and complicated functions of electron energy $(E)$ and field frequency $(\omega)$. Finally, the wave function of electron-field system is definitely fixed. In its turn, according to the quantum mechanics [30], it determines the stationary and dynamic densities of electronic current through the RTS. The stationary densities of input and output current define the transmission coefficient

$$
D(E)=\left|A_{08}^{(+)}(E) / A_{00}^{(+)}(E)\right|^{2},
$$

which, in its turn, fixes the spectral parameters (resonance energies $E_{n}$ and widths $\Gamma_{n}$ ) of electron quasistationary states [17].

The calculation of dynamic currents $[18,20]$ makes it possible to obtain the real part of dynamic conductivity of the system caused by the electron-field interaction. It is convenient to express this conductivity as a sum of two partial terms

$$
\sigma(E, \omega)=\sigma^{+}(E, \omega)+\sigma^{-}(E, \omega) .
$$

Herein

$$
\begin{aligned}
& \sigma^{ \pm}(E, \omega)=\frac{\hbar^{2} \omega n_{0}}{2 d m_{\mathrm{w}} F^{2}} \\
& \times\left(k_{+1}\left|A_{+1\left\{\begin{array}{l}
8 \\
0
\end{array}\right\}}^{( \pm)}(E, \omega, F)\right|^{2}-k_{-1}\left|A_{-1\left\{\begin{array}{l}
8 \\
0
\end{array}\right\}}^{( \pm)}(E, \omega, F)\right|^{2}\right),
\end{aligned}
$$

are the partial terms caused by the input $\left(\sigma^{+}\right)$and output $\left(\sigma^{-}\right)$electronic current through the RTS, respectively. As far as the coefficients $\left(A^{( \pm)}\right)$are the linear functions of the field intensity, expressions (12), (14), both $D$ and $\sigma$ are independent of it in the framework of small signal onemode approximation. The transmission coefficient $(D)$ is the function of electron energy $(E)$ while the dynamic conductivity $(\sigma)$ is proportional to the concentration $\left(n_{0}\right)$ and depends both on the electron energy $(E)$ and field frequency $(\omega)$. Both of them depend on geometrical parameters of three-well RTS.

\section{Hamiltonian of electron-phonon system in closed three-well RTS in the representation of second quantization}

In order to develop the theory of electron-phonon interaction in open multi-layer RTS within the Green functions approach, one has to use the complete set of orthonormal electron wave functions. These functions for the quasi-stationary states corresponding to the underbarrier resonance energies $(E<U)$ should be localized enough. It is clear that the final results are to be independent of the choice of the set of functions. However, this choice determines the character of the problems arising in the approach analyzed in detail in Ref. [28]. The spread method is to assume that the thicknesses of RTS outer barriers are rather big and, thus, the barriers become infinite. Consequently, the electron under-barrier states are characterized by localized wave functions obtained from the stationary Schrödinger equation and the abovebarrier states - by the non-localized ones.

According to the abovementioned, we are going to study the closed (c) three-well RTS with infinite outer barriers ( $b_{1}=b_{4} \rightarrow \infty$, Fig. 1$)$. Thus, in the Hamiltonian (2), instead of the potential energy $U_{\mathrm{e}}^{(\mathrm{o})}\left(z, b_{1}, b_{4}\right)$ for open RTS we put $U_{\mathrm{e}}^{(\mathrm{c})}=\lim _{b_{1}, b_{4} \rightarrow \infty} U_{\mathrm{e}}^{(\mathrm{o})}\left(z, b_{1}, b_{4}\right)$ for the closed one.

The solution of stationary Schrödinger equation with Hamiltonian (1) for the electron in closed three-well RTS is written as

$$
\begin{aligned}
& \Psi_{\tilde{n} \boldsymbol{k}}^{(\mathrm{c})}(\boldsymbol{r})=\Psi_{\tilde{n} \boldsymbol{k}}^{(\mathrm{c})}(\boldsymbol{\rho}, z)=\frac{1}{\sqrt{S}} \mathrm{e}^{\mathrm{i} \boldsymbol{k} \boldsymbol{\rho}} \Psi_{\tilde{n}}^{(\mathrm{c})}(z), \\
& \boldsymbol{k}=\boldsymbol{n}_{x} k_{x}+\boldsymbol{n}_{y} k_{y}, \quad \tilde{n}=\left\{\begin{array}{ll}
k_{\perp}, & E \geq U \\
n, & E<U
\end{array}\right\},
\end{aligned}
$$

where $S$ is the square of the main region in $X O Y$ plane. We introduce the correlated effective mass $m_{\tilde{n}}^{-1}=$ $\int_{-\infty}^{\infty} \mathrm{d} z\left|\Psi_{\tilde{n}}^{(\mathrm{c})}(z)\right|^{2} / m(z)$ instead of $m(z)$ in the term $T_{\boldsymbol{\rho}}$, as in Ref. [31], in order to simplify the Hamiltonian $H_{\mathrm{e}}$ and separate the variables $\rho$ and $z$. As a result, the complete electron energy $E_{\tilde{n} \boldsymbol{k}}^{(\mathrm{c})}$ consists of kinetic energy of its movement in $X O Y$ plane $\left(\hbar^{2} k^{2} / 2 m_{\tilde{n}}\right)$ and the energy of longitudinal movement $E_{\tilde{n}}^{(\mathrm{c})}$ :

$$
E_{\tilde{n} \boldsymbol{k}}^{(\mathrm{c})}=\frac{\hbar^{2} \boldsymbol{k}^{2}}{2 m_{\tilde{n}}}+E_{\tilde{n}}^{(\mathrm{c})} .
$$

The energy spectrum $\left(E_{\tilde{n}}^{(\mathrm{c})}\right)$ and wave functions $\left(\Psi_{\tilde{n}}^{(\mathrm{c})}(z)\right)$ are obtained from the one-dimensional Schrödinger equation 


$$
\begin{aligned}
& \left\{-\frac{\hbar^{2}}{2} \frac{\mathrm{d}}{\mathrm{d} z} \frac{1}{m(z)} \frac{\mathrm{d}}{\mathrm{d} z}+U(z)\right\} \Psi_{\tilde{n}}^{(\mathrm{c})}(z)= \\
& E_{\tilde{n}}^{(\mathrm{c})} \Psi_{\tilde{n}}^{(\mathrm{c})}(z) .
\end{aligned}
$$

Its exact solutions are the functions

$$
\Psi_{\tilde{n}}^{(\mathrm{c})}(z)=\left\{\begin{array}{l}
\sum_{j=2,4,6} \Psi_{j \tilde{n}}^{(\mathrm{c})}(z)= \\
\sum_{j=2,4,6}\left(A_{j \tilde{n}}^{+} \mathrm{e}^{\mathrm{i} k_{\tilde{n}} z}+A_{j \tilde{n}}^{-} \mathrm{e}^{-\mathrm{i} k_{\tilde{n}} z}\right), \\
\sum_{j=1,3,5,7} \Psi_{j \tilde{n}}^{(\mathrm{c})}(z)= \\
\sum_{j=1,3,5,7}\left(A_{j \tilde{n}}^{+} \mathrm{e}^{\chi_{\tilde{n}} z}+A_{j \tilde{n}}^{-} \mathrm{e}^{-\chi_{\tilde{n}} z}\right),
\end{array}\right.
$$

where

$$
\begin{aligned}
& k_{\tilde{n}}=\hbar^{-1} \sqrt{2 m_{\mathrm{w}} E_{\tilde{n}}^{(\mathrm{c})}}, \\
& \chi_{\tilde{n}}=\hbar^{-1} \sqrt{2 m_{\mathrm{b}}\left(U-E_{\tilde{n}}^{(\mathrm{c})}\right)}=\sqrt{2 m_{\mathrm{b}} U \hbar^{-2}-k_{\tilde{n}}^{2} m_{\mathrm{b}} / m_{\mathrm{w}}} .
\end{aligned}
$$

The fitting conditions

$$
\begin{aligned}
& \left.\Psi_{j \tilde{n}}^{(\mathrm{c})}(z)\right|_{z=z_{j}}=\left.\Psi_{j+1 \tilde{n}}^{(\mathrm{c})}(z)\right|_{z=z_{j}} ; \\
& \left.\quad \frac{\partial \Psi_{j \tilde{n}}^{(\mathrm{c})}(z)}{m_{j}(z) \partial z}\right|_{z=z_{j}}=\left.\frac{\partial \Psi_{j+1 \tilde{n}}^{(\mathrm{c})}(z)}{m_{j+1}(z) \partial z}\right|_{z=z_{j}}, \quad j=1-6
\end{aligned}
$$

and normalization ones

$$
\begin{aligned}
& \int_{-\infty}^{\infty} \Psi_{n}^{(\mathrm{c}) *}(z) \Psi_{n^{\prime}}^{(\mathrm{c})}(z) \mathrm{d} z=\delta_{n n^{\prime}}, \\
& \int_{-\infty}^{\infty} \Psi_{k_{\perp}^{\prime}}^{(\mathrm{c}) *}(z) \Psi_{k_{\perp}}^{(\mathrm{c})}(z) \mathrm{d} z=\delta\left(k_{\perp}-k_{\perp}^{\prime}\right)
\end{aligned}
$$

definitely fix the coefficients $\left(A_{j \tilde{n}}^{ \pm}\right)$, wave functions $\left(\Psi_{j \tilde{n}}^{(\mathrm{c})}(z)\right)$, energies $\left(E_{\tilde{n}}^{(\mathrm{c})}\right)$ and, since, the complete energy spectrum $\left(E_{\tilde{n} k}^{(\mathrm{c})}\right)$ and orthonormalized set of wave functions $\left(\Psi_{\tilde{n} k}^{(\mathrm{c})}(\rho, z)\right)$.

Introducing the quantized wave function

$$
\Psi(\boldsymbol{\rho}, z)=\sum_{\tilde{n}, \boldsymbol{k}} \Psi_{\tilde{n} \boldsymbol{k}}^{(\mathrm{c})}(\boldsymbol{\rho}, z) a_{\tilde{n} \boldsymbol{k}}
$$

with creation $\left(a_{\tilde{n} \boldsymbol{k}}^{+}\right)$and annihilation $\left(a_{\tilde{n} \boldsymbol{k}}\right)$ Fermi operators of electron states, we obtain the Hamiltonian (1) in the representation of their occupation numbers

$$
\begin{aligned}
& H_{\mathrm{e}}=\iint \Psi^{+}(\boldsymbol{\rho}, z) H_{\mathrm{e}}(\boldsymbol{\rho}, z) \Psi(\boldsymbol{\rho}, z) \mathrm{d} \boldsymbol{\rho} \mathrm{d} z= \\
& \sum_{\tilde{n} \boldsymbol{k}} E_{\tilde{n} \boldsymbol{k}}^{(\mathrm{c})} a_{\tilde{n} \boldsymbol{k}}^{+} a_{\tilde{n} \boldsymbol{k}} .
\end{aligned}
$$

It is well known [23-29], that in the dielectric continuum model, the spectra and potentials of polarization fields of optical confined $\left(\Phi_{\mathrm{L}}(r)\right)$ and interface $\left(\Phi_{\mathrm{I}}(r)\right)$ phonons are obtained from the following equation:

$$
\varepsilon_{j}(\omega) \nabla^{2} \Phi(r)=0 .
$$

Herein, for the nanostructure with ternary compound $j$-th layer, the dielectric functions $\left(\varepsilon_{j}(\omega)\right)$ are fixed by the Lyddane-Sachs-Teller relation

$$
\varepsilon_{j}(\omega)=\varepsilon_{j \infty} \frac{\left(\omega^{2}-\omega_{j L_{1}}^{2}\right)\left(\omega^{2}-\omega_{j L_{2}}^{2}\right)}{\left(\omega^{2}-\omega_{j T_{1}}^{2}\right)\left(\omega^{2}-\omega_{j T_{2}}^{2}\right)},
$$

where $\varepsilon_{j \infty}$ is high frequency dielectric constant, $\omega_{j L_{1}}$, $\omega_{j L_{2}}$ and $\omega_{j T_{1}}, \omega_{j T_{2}}$ are the frequencies of longitudinal $\left(L_{j 1}, L_{j 2}\right)$ and transversal $\left(T_{j 1}, T_{j 2}\right)$ phonon modes of the respective bulk crystals.

Solving Eq. (24) and using the known [29] procedure of quantization of confined phonons field $\left(\Phi_{\mathrm{L}}(r)\right)$ at $\varepsilon_{j}(\omega)=0, \nabla^{2} \Phi_{\mathrm{L}}(r) \neq 0$ and interface phonons field $\left(\Phi_{\mathrm{I}}(r)\right)$ at $\varepsilon_{j}(\omega) \neq 0, \nabla^{2} \Phi_{\mathrm{I}}(r)=0$, we obtain the Hamiltonian of phonon system

$$
\begin{aligned}
& H_{\mathrm{ph}}=H_{\mathrm{L}}+H_{\mathrm{I}}=\sum_{j=1}^{7} \sum_{l=1}^{2} \sum_{\lambda q} \Omega_{j L_{l}}\left(b_{j L_{l} \lambda q}^{+} b_{j L_{l} \lambda q}+1 / 2\right) \\
& \quad+\sum_{s q} \Omega_{I s q}\left(b_{I s q}^{+} b_{I s q}+1 / 2\right),
\end{aligned}
$$

where $\Omega_{j L_{l}}=\hbar \omega_{j L_{l}}$ are the energies and $b_{j L_{l} \lambda q}, b_{j L_{l} \lambda q}^{+}$ are Bose operators of the pairs $(l=1,2)$ of confined phonons of $j$-th layer; $\Omega_{I s q}$ are the energies and $b_{I s q}, b_{I s q}^{+}$are Bose operators of interface phonons where $s$ means the number of mode with two-dimensional quasimomentum $q$. The energies of interface phonons are obtained from the dispersion equation

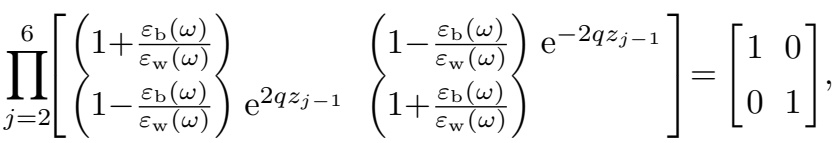

found from the condition of non-trivial solution of the system of equations

$$
\left.\begin{array}{l}
f_{j} \mathrm{e}^{-q z_{j}}+g_{j} \mathrm{e}^{q z_{j}}=f_{j+1} \mathrm{e}^{-q z_{j}}+g_{j+1} \mathrm{e}^{q z_{j}} \\
\varepsilon_{j}(\omega)\left[f_{j} \mathrm{e}^{-q z_{j}}-g_{j} \mathrm{e}^{q z_{j}}\right]= \\
\varepsilon_{j+1}(\omega)\left[f_{j+1} \mathrm{e}^{-q z_{j}}-g_{j+1} \mathrm{e}^{q z_{j}}\right]
\end{array}\right\}(j=1-5),
$$

fixing the continuity of induction and polarization field of $I$-phonons at all nanostructure interfaces.

The Hamiltonian of electron-phonon interaction $H_{\mathrm{e}-\mathrm{ph}}(\boldsymbol{r})=-e\left(\Phi_{\mathrm{L}}(\boldsymbol{r})+\Phi_{\mathrm{I}}(\boldsymbol{r})\right)$ after the normalization and quantization of both fields (L- and I-phonons) and transition from the coordinate representation to the occupation numbers one, using the quantized wave function (22), is obtained in the representation of second quantization over all variables of the system

$$
H_{\mathrm{e}-\mathrm{ph}}=H_{\mathrm{e}-\mathrm{L}}+H_{\mathrm{e}-\mathrm{I}}=
$$

$$
\begin{aligned}
& \sum_{j=1}^{7} \sum_{l=1}^{2} \sum_{\lambda q} \sum_{\tilde{n}_{1} \tilde{n} k} F_{\tilde{n}_{1} \tilde{n}}^{j L_{l}}(\lambda, q) a_{\tilde{n}_{1} k+q}^{+} a_{\tilde{n} k}\left(b_{j L_{l} \lambda, q}+b_{j L_{l} \lambda,-q}^{+}\right) \\
& +\sum_{s q} \sum_{\tilde{n}_{1} \tilde{n} k} F_{\tilde{n}_{1} \tilde{n}}^{I}(s, q) a_{\tilde{n}_{1} k+q}^{+} a_{\tilde{n} k}\left(b_{s, q}+b_{s,-q}^{+}\right) .
\end{aligned}
$$




$$
\begin{aligned}
& F_{\tilde{n}_{1} \tilde{n}}^{j L_{l}}(\lambda, q)=-\sqrt{\frac{8 \pi e^{2} \hbar}{S\left[\pi^{2} \lambda^{2}+q^{2}\left(z_{j}-z_{j-1}\right)^{2}\right]}}\left[\left.\frac{\partial \varepsilon_{j}(\omega)}{\partial \omega}\right|_{\omega=\omega_{j} L_{l}}\right]^{-1 / 2} \int_{z_{j-1}}^{z_{j}} \mathrm{~d} z \Psi_{j \tilde{n}_{1} *}^{(c) *}(z) \Psi_{j \tilde{n}}^{(\mathrm{c})}(z) \\
& \quad \times\left\{\begin{array}{l}
\left.\cos \left[\pi \lambda\left(\frac{z-z_{j-1}}{z_{j}-z_{j-1}}-\frac{1}{2}\right)\right]\right|_{\lambda=1,3,5 \ldots} \\
\left.\sin \left(\pi \lambda\left(\frac{z-z_{j-1}}{z_{j}-z_{j-1}}-\frac{1}{2}\right)\right)\right|_{\lambda=2,4,6 \ldots}
\end{array} \quad j=1-7, z_{-1} \rightarrow-\infty, z_{7} \rightarrow \infty\right.
\end{aligned}
$$

- the binding functions of electrons in states $\tilde{n}_{1}, \tilde{n}$ with L-phonons of $j$-th layer,

$$
\begin{aligned}
& F_{\tilde{n}_{1} \tilde{n}}^{I}(s, q)=-\sqrt{\frac{4 \pi e^{2} \hbar}{q S N(s, q)}} \sum_{j=0}^{7} \int_{z_{j-1}}^{z_{j}} \mathrm{~d} z \Psi_{\tilde{n}_{1}}^{(\mathrm{c}) *}(z) \Psi_{\tilde{n}}^{(\mathrm{c})}(z) \\
& \quad \times\left[f_{j}(s, q) \mathrm{e}^{-q z}+g_{j}(s, q) \mathrm{e}^{q z}\right]
\end{aligned}
$$

- the binding functions of electrons in states $\tilde{n}_{1}, \tilde{n}$ with I-phonons. The normalization coefficient

$$
\begin{aligned}
& N(s, q)=\left.\sum_{j=0}^{7} \frac{\partial \varepsilon_{j}(\omega)}{\partial \omega}\right|_{\omega=\omega_{s q}}\left[g_{j}^{2}(s, q)\left(\mathrm{e}^{2 q z_{j}}-\mathrm{e}^{2 q z_{j-1}}\right)\right. \\
& \left.-f_{j}^{2}(s, q)\left(e^{-2 q z_{j}}-\mathrm{e}^{-2 q z_{j-1}}\right)\right]
\end{aligned}
$$

is determined by the magnitudes $f_{j}, g_{j}$, obtained from the system of Eqs. (28). The integrals in the formulae (30), (31) are analytically calculated but we do not present them due to their sophisticated forms.

The obtained Hamiltonian of electron-phonon system in three-well RTS in the representation of second quantization over all variables

$$
H=H_{\mathrm{e}}+H_{\mathrm{L}}+H_{\mathrm{I}}+H_{\mathrm{e}-\mathrm{L}}+H_{\mathrm{e}-\mathrm{I}},
$$

allows us to calculate the Fourier-image of electron Green functions for the discrete spectrum $(\tilde{n}=n)$.

At finite temperature $(T \neq 0 \mathrm{~K})$, small concentration of electrons and weak binding with phonons, according to the rules of the Feynman-Pines diagram technique [32], the Fourier-image of electron Green function is found from Dyson equation

$$
G_{n}(\boldsymbol{k}, \hbar \omega)=\left\{\hbar \omega-E_{n k}^{(\mathrm{c})}-M_{n}(\hbar \omega, \boldsymbol{k})\right\}^{-1}
$$

with mass operator (MO) $M_{n}(\hbar \omega, \boldsymbol{k})$, calculated in onephonon approximation taking into account the discrete $\left(\tilde{n}_{1}=n_{1}\right)$ and continuum $\left(\tilde{n}_{1}=k_{\perp}\right)$ parts of the spectrum

$$
\begin{gathered}
M_{n}(\hbar \omega, k)=\sum_{\tilde{n}_{1}, p, q} F_{n \tilde{n}_{1}}^{*}(p, q) F_{\tilde{n}_{1} n}(p, q) \\
\times\left[\frac{1+\nu_{p q}}{\hbar \omega-E_{\tilde{n}_{1}}^{(\mathrm{c})}(k-q)-\Omega_{p q}+\mathrm{i} \eta}\right. \\
\left.+\frac{\nu_{p q}}{\hbar \omega-E_{\tilde{n}_{1}}^{(\mathrm{c})}(k+q)+\Omega_{p q}+\mathrm{i} \eta}\right] .
\end{gathered}
$$

Here we introduced the generalized index for the phonon modes $(p=\{j, \ell, \lambda ; s\})$, which at $p=j, \ell, \lambda$ numerates all modes of L-phonons in $j$-th layers of three-well RTS and at $p=s-$ all I-phonon modes, respectively. The magnitudes $\nu_{p q}=\left\{\mathrm{e}^{\Omega_{p q} / k_{B} T}-1\right\}^{-1}$ are the average phonon occupation numbers for the respective modes. The first term in MO (35) describes the physical processes accompanied by the creation of phonons and second one - by the annihilation.

According to the theory of the Green functions [32], at weak electron-phonon interaction, the discrete part of electron energy spectrum $\left(E_{n}^{(\mathrm{c})}\right)$ is renormalized $\left(\tilde{E}_{n}^{(\mathrm{c})}\right)$ due to the shift $\Delta_{n}$

$$
\begin{aligned}
& \tilde{E}_{n}^{(\mathrm{c})}=E_{n}^{(\mathrm{c})}+\Delta_{n}= \\
& E_{n}^{(\mathrm{c})}+\operatorname{Re} M_{n}\left(\hbar \omega=E_{n}^{(\mathrm{c})}, k=0\right)
\end{aligned}
$$

and decay rate

$$
\gamma_{n}=-2 \operatorname{Im} M_{n}\left(\hbar \omega=E_{n}^{(\mathrm{c})}, \boldsymbol{k}=0\right) .
$$

Further, we are going to study the contributions of different mechanisms of electron-phonon interaction into the complete shift of the energies $\left(\Delta_{n}\right)$ and decay rates $\left(\gamma_{n}\right)$ of $n$-th state. These parameters, according to the additive form of MO (35), are conveniently written as

$$
\begin{aligned}
& \Delta_{n}=\Delta_{n}^{L}+\Delta_{n}^{I}= \\
& \quad\left(\Delta_{n n}^{L}+\Delta_{n d}^{L}+\Delta_{n c}^{L}\right)+\left(\Delta_{n n}^{I}+\Delta_{n d}^{I}+\Delta_{n c}^{I}\right), \\
& \gamma_{n}=\gamma_{n}^{L}+\gamma_{n}^{I}=\left(\gamma_{n n}^{L}+\gamma_{n d}^{L}+\gamma_{n c}^{L}\right) \\
& \quad+\left(\gamma_{n n}^{I}+\gamma_{n d}^{I}+\gamma_{n c}^{I}\right),
\end{aligned}
$$

where $\Delta_{n n}^{L, I}, \gamma_{n n}^{L, I}$ are the partial contributions into the shift $\left(\Delta_{n}\right)$ and decay rate $\left(\gamma_{n}\right)$ produced by intraband electron-phonon interaction with L- and I-phonons; $\Delta_{n d}^{L, I}=\sum_{n_{1} \neq n} \Delta_{n n_{1}}^{L, I}, \gamma_{n d}^{L, I}=\sum_{n_{1} \neq n} \gamma_{n n_{1}}^{L, I}$ are the partial contributions produced by interband interaction with L- and I-phonons through the all states of discrete (d) spectrum; $\Delta_{n c}^{L, I}=\sum_{k_{\perp}} \Delta_{n k_{\perp}}^{L, I}, \gamma_{n c}^{L, I}=\sum_{k_{\perp}} \gamma_{n k_{\perp}}^{L, I}$ are the partial contributions produced by electron-phonon interaction through the all states of continuum (c).

The analytical calculation of partial shifts and decay rates is performed using the corresponding terms of MO (35) and transiting from sum over quasimomentum $\boldsymbol{q}$ to the integral $\left(\sum_{q} \Rightarrow S / 4 \pi^{2} \iint \mathrm{d}^{2} q\right)$ with Dirac relationship

$$
\begin{aligned}
& \iint \frac{\mathrm{d}^{2} \boldsymbol{q}}{f(\boldsymbol{q})}+\mathrm{i} \eta= \\
& P \iint \frac{\mathrm{d}^{2} \boldsymbol{q}}{f(\boldsymbol{q})}-\mathrm{i} \pi \iint \delta(f(\boldsymbol{q})) \mathrm{d}^{2} \boldsymbol{q},
\end{aligned}
$$


where symbol $P$ means that the respective integrals are taken as Cauchy principal values.

The partial shifts of the energy $\left(\Delta_{n n}\right)$ and decay rate $\left(\gamma_{n n}\right)$ of $n$-th state due to the intraband interaction with L- and ?? I-phonons are given by the expressions

$$
\Delta_{n n}=
$$

$$
\begin{gathered}
\frac{S}{4 \pi^{2}} \sum_{ \pm, p} P \iint \frac{\left|F_{n n}(p, q)\right|^{2}\left(\nu_{p q}+1 / 2 \pm 1 / 2\right)}{E_{n}^{(\mathrm{c})}-E_{n q}^{(\mathrm{c})} \pm \Omega_{p q}} \mathrm{~d}^{2} \boldsymbol{q} \\
\gamma_{n n}=\frac{S}{2 \pi} \sum_{ \pm, p} \iint\left|F_{n n}(p, q)\right|^{2}\left(\nu_{p q}+1 / 2 \pm 1 / 2\right) \\
\times \delta\left(E_{n}^{(\mathrm{c})}-E_{n q}^{(\mathrm{c})} \pm \Omega_{p q}\right) \mathrm{d}^{2} q .
\end{gathered}
$$

These expressions are exactly integrated analytically for the partial contributions produced by dispersionless Lphonons. The calculation of I-phonons contributions is performed within numeric computer integration because the occupation numbers $\left(\nu_{s q}\right)$ depend on quasimomentum $q$.

\section{Discussion of the results}

In order to study the temperature dependences of electromagnetic field absorption bands parameters (shifts and broadening) caused by quantum transitions between electron quasi-stationary states in active region of QCD one has to study the temperature dependences of spectral parameters (resonance energies and widths) of electron quasi-stationary states. The latter are mainly caused by the varying potential profile of RTS due to the varying width of energy gap for the wells and barriers and electron-phonon interaction within the temperature dependence of phonon occupation numbers because the nanostructures, grown by molecular beam epitaxy method, almost do not contain impurities and have rather perfect interfaces between nanolayers.

In order to study the effect of both abovementioned mechanisms at temperature dependence of electromagnetic field absorption band, according to the theory developed earlier, we calculated the electron energy spectrum $\left(E_{n}^{\mathrm{c}}\right)$ in closed model together with the spectral parameters (resonance energies $\left(E_{n}^{\mathrm{o}}\right)$, widths $\left(\Gamma_{n}^{\mathrm{o}}\right)$ ) and dynamic conductivity in open three-well RTS (without taking into account the electron-phonon interaction). The nanostructure is studied as expanded active region (EAR) of QCD separate cascade: in addition to two wells (layers 4 and 6 in Fig. 1), where the electrons are mainly located in operating quasi-stationary states $(n=1 ; 3)$ between which the quantum transition accompanied by absorption of electromagnetic field happens, it contains the last well (layer 2) of the extractor of previous cascade from which the electrons, emitting L- and I-phonons, get into the ground state of active region, being localized mainly in input well of active region (layer 4).

In order to compare the results of computer calculations with experimental data, we studied the three-well
RTS with $\operatorname{In}_{0.53} \mathrm{Ga}_{0.47}$ As wells and $\operatorname{In}_{0.52} \mathrm{Al}_{0.48}$ As barriers, creating the EAR of QCD separate cascade investigated in experimental paper [3]. Geometrical parameters of open RTS are taken the same as in [3]: $a_{1}=4.7 \mathrm{~nm}, a_{2}=5.9 \mathrm{~nm}, a_{3}=1.7 \mathrm{~nm}, b_{1}=2.8 \mathrm{~nm}$, $b_{2}=2.6 \mathrm{~nm}, b_{3}=6.0 \mathrm{~nm}, b_{4}=4.4 \mathrm{~nm}$. The effective masses of electrons in wells $\left(m_{\mathrm{w}}=0.051 m_{\mathrm{e}}\right)$ and barriers $\left(m_{\mathrm{b}}=0.084 m_{\mathrm{e}}\right)$ are assumed as constant and the heights of potential barriers are different $\left(U_{T=10 \mathrm{~K}}=554 \mathrm{meV}\right.$; $U_{T=300 \mathrm{~K}}=520 \mathrm{meV}$ ) due to the temperature dependences of the energy gaps [33]. We calculated the three lower energies of electron spectrum for the closed model and resonance energies and widths for the open three-well RTS at cryogenic $(T=10 \mathrm{~K})$ and room $(T=300 \mathrm{~K})$ temperatures (Table I). The results of calculations presented in Table I prove that in both models, the energies of two lower states are coinciding till the decimal numbers and the energy of the third one differs at $2-3$ percents. In the closed model the states are stationary, while in the open one the states are quasi-stationary with resonance widths $\left(\Gamma_{n}^{\mathrm{o}}\right)$.

TABLE I

Three lower energies of electron spectrum for the closed model and resonance energies and widths for the open three-well RTS at cryogenic $(T=10 \mathrm{~K})$ and room $(T=300 \mathrm{~K})$ temperatures.

\begin{tabular}{c|c|c|c|c}
\hline \hline$n$ & $T[\mathrm{k}]$ & $E_{n}^{\mathrm{c}}[\mathrm{meV}]$ & $E_{n}^{\mathrm{o}}[\mathrm{meV}]$ & $\Gamma_{n}[\mathrm{meV}]$ \\
\hline \multirow{2}{*}{1} & 10 & 93.64 & 93.14 & 0.007 \\
& 300 & 91.63 & 91.03 & 0.011 \\
\hline \multirow{2}{*}{2} & 10 & 125.53 & 126.08 & 0.601 \\
& 300 & 122.47 & 123.15 & 0.727 \\
\hline \multirow{2}{*}{3} & 10 & 324.73 & 334.06 & 8.667 \\
& 300 & 311.61 & 320.60 & 9.930
\end{tabular}

According to the general idea of experimental QCD [3], the electrons from the state $(n=2)$ being in extractor well (layer 2, Fig. 1) are emitting phonons and transit into the state $(n=1)$ of the well (layer 4 ) of the main element of active region (MEAR), where from, absorbing the electromagnetic field energy, within the quantum transition, the electrons get into the state $(n=3)$, being mainly located in the well (layer 6) of expanded active region (EAR). We studied the resonance energies $\left(E_{n}^{\mathrm{o}}\right)$, widths $\left(\Gamma_{n}^{\mathrm{o}}\right)$ and dynamic conductivities $\left(\sigma_{13}, \sigma_{13}^{ \pm}\right)$for the open model at $T=10 \mathrm{~K}$ and $T=300 \mathrm{~K}$ depending on the design of three-well RTS, being an EAR of QCD. For the fixed, as in paper [3], sizes of the well $\left(a_{2}=5.9 \mathrm{~nm}\right)$ and barriers $\left(b_{2}=2.6 \mathrm{~nm}, b_{3}=6.0 \mathrm{~nm}\right)$ of MEAR and sum of the sizes of outer wells $\left(a=a_{1}+a_{3}=6.4 \mathrm{~nm}\right.$, $\left.a_{1}=4.7 \mathrm{~nm}, a_{3}=1.7 \mathrm{~nm}\right)$, we calculated $E_{n}^{\mathrm{o}}, \Gamma_{n}^{\mathrm{o}}$, $\sigma_{13}, \sigma_{13}^{ \pm}$as functions of the position of MEAR between the outer barriers (layers 1 and 7 ) of EAR, that is of the varying size $\left(0 \leq a_{1} \leq a\right)$ of the well (layer 2$)$.

As far as spectral parameters and dynamic conductivities depend on $a_{1}$ qualitatively and quantitatively similarly at both temperatures, in Fig. 2 we present $E_{n}^{\mathrm{o} T}$, 


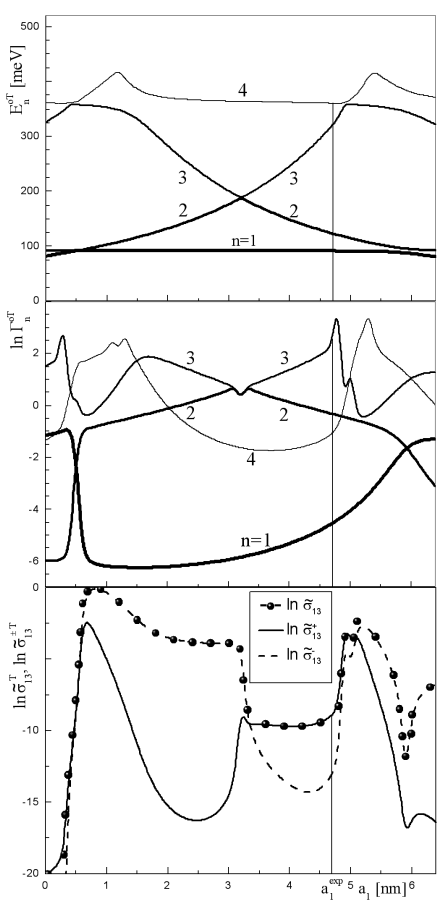

Fig. 2. Spectral parameters of electron quasistationary states, complete and partial terms of dynamic conductivity as functions of the position of main element of active region $\left(a_{1}\right)$ in three-barrier RTS at $T=300 \mathrm{~K}\left(a_{2}=5.9 \mathrm{~nm}, a=6.4 \mathrm{~nm}, \Delta_{1}=2.8 \mathrm{~nm}\right.$, $\left.\Delta_{2}=2.6 \mathrm{~nm}, \Delta_{3}=6 \mathrm{~nm}, \Delta_{4}=2.8 \mathrm{~nm}\right)$.

$\ln \Gamma_{n}^{\mathrm{oT}}, \ln \tilde{\sigma}_{13}^{T}, \ln \tilde{\sigma}_{13}^{ \pm T}$ only at $T=300 \mathrm{~K}$. Herein, the dynamic conductivities are conveniently expressed in relative units $\tilde{\sigma}_{13}=\left(\sigma_{13} / \max \sigma_{13}\right)$, where $\max \sigma_{13}$ is the maximal magnitude of $\sigma_{13}$ in the range $0 \leq a_{1} \leq a$. Figure proves that design of three-well RTS, i.e. the position $\left(a_{1}\right)$ of MEAR in EAR, brings to the varying of resonance energies: weak for $E_{1}^{\mathrm{o}}$ and $E_{4}^{\mathrm{o}}$ (till $10 \%$ ) and considerable one for $E_{2}^{o}$ and $E_{3}^{o}$ (twice). It is caused by the fact that the ground and fourth quasi-stationary state are formed by the well (layer 4) and barriers (layers 3 and 5) of MEAR with fixed sizes while second and third ones are formed by the wells (layers 2 and 6 ) of EAR with varying sizes.

The resonance widths $\left(\Gamma_{n}^{\circ}\right)$ of these four quasistationary states are strongly non-linear functions of $a_{1}$, because the life times (decay rates) of these states depend on the probability of electron location in definite well of RTS, as well as on the thicknesses of the barriers through which it tunnels.

Partial terms of dynamic conductivity are formed by forward $\left(\sigma_{13}^{+}\right)$and backward $\left(\sigma_{13}^{-}\right)$electronic currents through the RTS, thus, as one can see from Fig. 2, their magnitudes and, since, the complete dynamic conductivity $\left(\sigma_{13}\right)$ strongly depend on the fact, where the electron is located with bigger probability, as a result of quantum transition: in the input (layer 2) or output (layer 6) well of EAR. The configuration of EAR in the experimental QCD [3] is realized in such a way $\left(a_{1}^{\exp }=4.7 \mathrm{~nm}\right.$, $\left.a_{3}^{\exp }=1.7 \mathrm{~nm}\right)$ that in the quantum transition from the first quasi-stationary state formed by the well (layer 4 ) of MEAR into the third one formed by output well (layer 6) the dynamic conductivity $\left(\sigma_{13}\right)$ appears. It is, mainly, formed by forward current, which is much bigger than the backward one $\left(\sigma_{13}^{+} \approx 55 \sigma_{13}^{-}\right)$. It ensures the tunneling of electrons into the next extractor of the cascade and, since, the QCD operation.

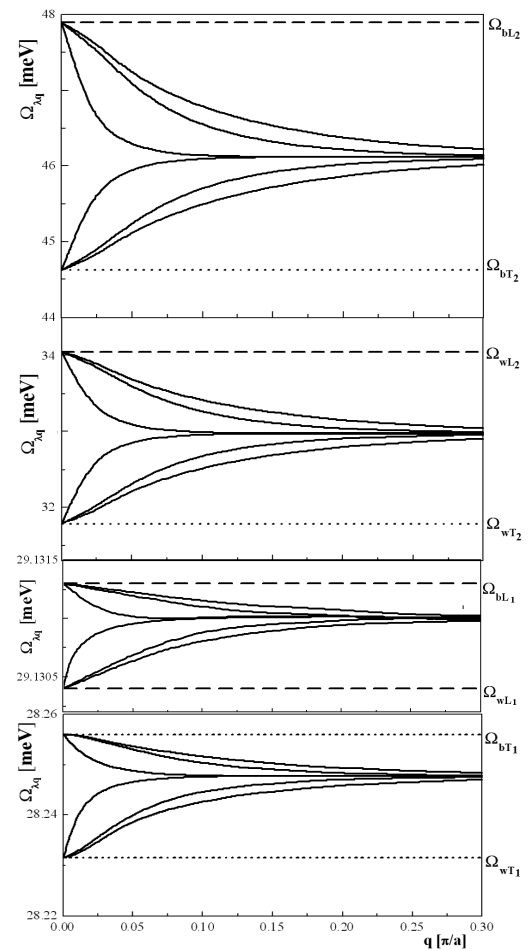

Fig. 3. Energy spectra of confined optical L-phonons and I-phonons in three-well RTS with ternary compound layers.

The effect of L- and I-phonons on electron spectrum is studied for the closed three-well RTS $\left(\mathrm{In}_{0.53} \mathrm{Ga}_{0.47} \mathrm{As} / \mathrm{In}_{0.52} \mathrm{Al}_{0.48} \mathrm{As}\right)$ using the dielectric continuum model with the same sizes of EAR wells and barriers as in [3] and physical parameters $\varepsilon_{0 w}=14.21$, $\varepsilon_{\infty \mathrm{w}}=11.63, \varepsilon_{0 \mathrm{~b}}=12.73, \varepsilon_{\infty \mathrm{b}}=8.15 ; \Omega_{\mathrm{w} L_{1}}=$ $29.1313 \mathrm{meV}, \Omega_{\mathrm{w} L_{2}}=34.05 \mathrm{meV}, \Omega_{\mathrm{b} L_{1}}=29.1304 \mathrm{meV}$, $\Omega_{\mathrm{b} L_{2}}=47.8905 \mathrm{meV}$. The I-phonons spectrum obtained from dispersion Eq. (27) and confined L-phonons are presented in Fig. 3. Here one can see 4 modes of confined L-phonons, which are dispersionless, and 24 modes of I-phonons with weak dispersion. The latter create 4 groups, containing 6 modes each. For the small quasimomentum each group contains three modes with positive and the three with negative dispersion, respectively. The two almost degenerated low-energy groups of I-phonon energies are placed between the energies of transversal $\left(\Omega_{\mathrm{w} T_{1}}, \Omega_{\mathrm{b} T_{1}}\right)$ and longitudinal $\left(\Omega_{\mathrm{b} L_{1}}, \Omega_{\mathrm{w} L_{1}}\right)$ phonons of the respective bulk crystals, while two highenergy groups with essential dispersion - between the energies $\left(\Omega_{\mathrm{w} T_{2}}, \Omega_{\mathrm{w} L_{2}}\right)$ and $\left(\Omega_{\mathrm{b} T_{2}}, \Omega_{\mathrm{b} L_{2}}\right)$. 
In order to study the effect of different phonon modes on the renormalized electron spectrum at cryogenic $(T=$ $10 \mathrm{~K})$ and room $(T=300 \mathrm{~K})$ temperatures, we calculated the complete shifts $\left(\Delta_{n}\right)$ and decay rates $\left(\gamma_{n}\right)$ together with their partial terms caused by the interaction between electrons and L-, I-phonons through the intraband states $\left(\Delta_{n n}, \gamma_{n n}\right)$ and due to the interband interaction through the states of discrete spectrum $\left(\Delta_{n d}, \gamma_{n d}\right)$ and continuum $\left(\Delta_{n c}, \gamma_{n c}\right)$. The results are presented in Table II for the three lower (operating) electron states. Here symbol $\Sigma$ means the sum contribution of L- and I-phonons into the spectral parameter.

TABLE II

The shifts and decay rates caused by the interaction between electrons and L-, I-phonons at cryogenic $(T=10 \mathrm{~K})$ and room $(T=300 \mathrm{~K})$ temperatures.

\begin{tabular}{|c|c|c|c|c|c|c|c|c|c|}
\hline \multirow[t]{2}{*}{$n$} & \multirow{2}{*}{$\begin{array}{c}T \\
{[\mathrm{~K}]}\end{array}$} & & $\Delta_{n n}$ & $\Delta_{n d}$ & $\Delta_{n c}$ & $\Delta_{n}$ & $\gamma_{n n}$ & $\gamma_{n d}$ & $\gamma_{n}$ \\
\hline & & & \multicolumn{7}{|c|}{$[\mathrm{meV}]$} \\
\hline \multirow{6}{*}{1} & & $\mathrm{~L}$ & 4 & 7 & 9 & -0.74 & 0 & , & 0 \\
\hline & & I & 68 & .10 & .003 & 3.78 & & & 0 \\
\hline & & $\Sigma$ & .32 & .17 & .032 & -4.52 & 0 & 0 & 0 \\
\hline & \multirow{3}{*}{300} & $\mathrm{~L}$ & -1.05 & -0.14 & -0.053 & -1.24 & .301 & .001 & .302 \\
\hline & & I & 3.60 & -0.29 & -0.015 & -3.91 & .001 & 97 & 1.098 \\
\hline & & $\Sigma$ & -4.65 & -0.43 & -0.068 & -5.15 & 1.302 & 0.098 & 1.400 \\
\hline \multirow{6}{*}{2} & & $\mathrm{~L}$ & 0.53 & -0.05 & -0.021 & -0.60 & 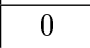 & - & 0 \\
\hline & 10 & $\mathrm{I}$ & 4.27 & .11 & -0.004 & -4.38 & 0 & 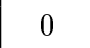 & 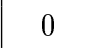 \\
\hline & & $\Sigma$ & 4.80 & -0.16 & -0.025 & -4.98 & & & 0 \\
\hline & & $\mathrm{L}$ & -0.90 & -0.08 & -0.053 & -1.03 & 0.227 & 0.001 & 0.228 \\
\hline & 300 & $\mathrm{I}$ & -4.12 & -0.44 & -0.021 & -4.58 & 1.197 & 0.047 & 1.244 \\
\hline & & $\Sigma$ & -5.02 & -0.52 & -0.074 & -5.61 & 1.425 & 0.048 & 1.473 \\
\hline & \multirow{3}{*}{10} & $\mathrm{~L}$ & .16 & .06 & .034 & -0.25 & 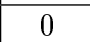 & 024 & .024 \\
\hline & & I & -4.60 & -0.34 & -0.017 & -4.96 & 0 & 53 & 0.053 \\
\hline & & $\Sigma$ & -4.76 & -0.40 & -0.0 & -5.21 & & 77 & 0.077 \\
\hline & \multirow{3}{*}{300} & $\mathrm{~L}$ & -0.28 & -0.10 & -0.051 & -0.43 & 0.079 & 0.016 & 0.095 \\
\hline & & I & -6.60 & -0.04 & -0.049 & -6.69 & 1.918 & 0.001 & 1.919 \\
\hline & & $\Sigma$ & -6.88 & -0.14 & -0.100 & -7.12 & 1.997 & 0.017 & 2.014 \\
\hline
\end{tabular}

The analysis of Table II proves that independently of the temperature, the spectral parameters of operating electron states $(n=1,2,3)$ are renormalized, mainly, due to electron-I-phonon interaction. Its contribution into $\Delta_{n}, \gamma_{n}$ is several times or the order bigger than that of L-phonons. The main contribution into the negative shifts $\left(\Delta_{n}<0\right)$ of all energy levels is performed by intraband $\left(\Delta_{n n}\right)$ interaction between electrons and L-, I-phonons. The contributions of interband interaction through the states of discrete spectrum $\left(\Delta_{n d}\right)$ are an order smaller and that of continuum $\left(\Delta_{n c}\right)$ are two orders smaller. The negative energy shifts $\left(\Delta_{n}\right)$ increase for the bigger $n$ and temperature.

The decay rates $\left(\gamma_{n}\right)$ of electron states at cryogenic temperature $T=10 \mathrm{~K}$ are mainly formed due to the interaction with virtual phonons in the processes of their creation because their average occupation number is very small $\left(\nu \approx 10^{-18}\right)$. Thus, according to the law of energy conservation, the decay rate of electron state $(n)$ produced by the intra- and interband interactions through the states with bigger energy $\left(n^{\prime}>n\right)$ is absent and the one produced by the interband interaction through the states with smaller energy $\left(n^{\prime}<n\right)$ happens at the condition $E_{n}^{(\mathrm{c})}(k)-E_{n^{\prime}}^{(\mathrm{c})}(k-q)-\Omega_{\lambda}(q)=0$. For the same reason, $\gamma_{1}=\gamma_{2}=0 ; \gamma_{3}=\gamma_{3 d}=\gamma_{31} \neq 0$ at $T=10 \mathrm{~K}$ (Table II).

At finite temperatures $(T=300 \mathrm{~K})$ the decay rates $\left(\gamma_{n}\right)$ of electron states are proportional to the average occupation numbers $\left(\nu_{\lambda q}\right)$ of real L- and I-phonons and are formed, mainly, in the processes of their annihilation. Table II proves that the main contribution into $\gamma_{n}$ is produced by intraband interaction with phonons, herein the contribution of I-phonons prevails over that of L-phonons.

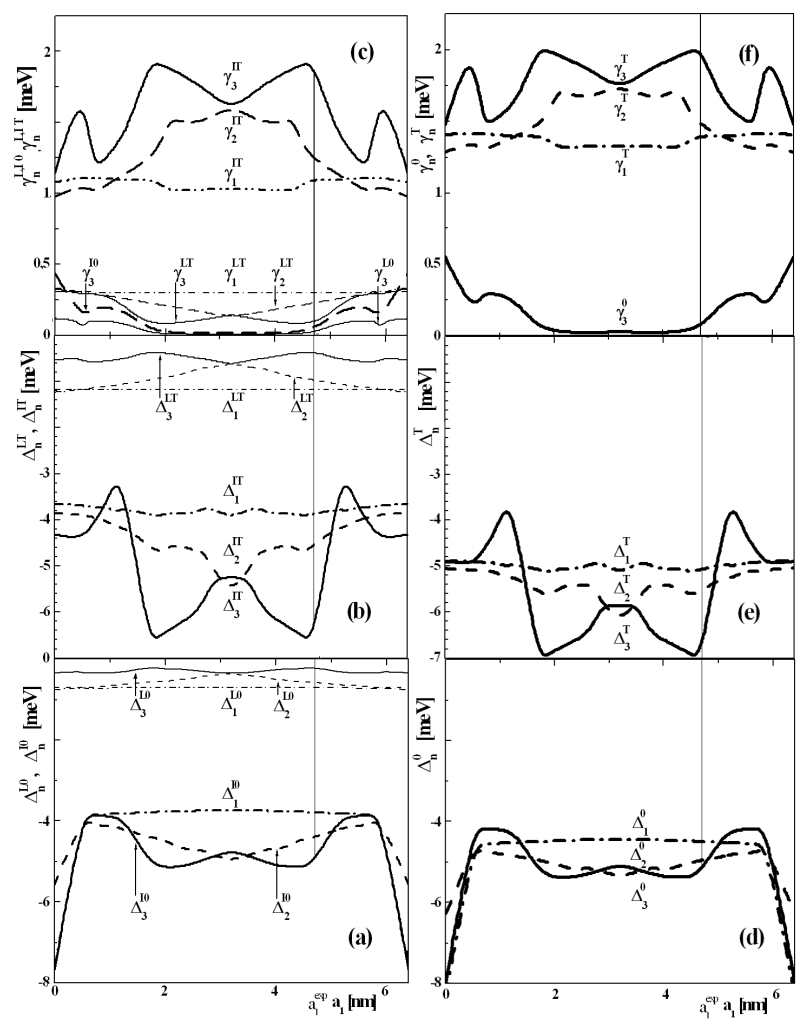

Fig. 4. The contributions of L- and I-phonons into the partial energy shifts and decay rates $(a, b, c)$ and complete energy shifts and decay rates $(\mathrm{d}, \mathrm{c}, \mathrm{f})$ of three operating states as functions of the position of main element of active region (the well width $\left(a_{1}\right)$ ) at $T=10 \mathrm{~K}$ ("0") and $T=300 \mathrm{~K}$ ("T").

In Fig. 4 the partial and complete energy shifts and decay rates of three operating electron states are presented as functions of position of MEAR $\left(a_{1}\right)$ in EAR at $T=10 \mathrm{~K}$ and $T=300 \mathrm{~K}$. One can see that the energy shifts and decay rates of different states nonlinearly depend on $a_{1}$, but the hierarchy of contributions of different mechanisms of electron-L- and I-phonon interactions is almost the same as previously analyzed one for the experimental configuration [3] of EAR at $a_{1}^{\exp }=4.7 \mathrm{~nm}$. 
TABLE III

Energies and widths of absorption bands for quantum transition $1 \rightarrow 3$ and differences between the energies of second and first state without taking into account phonons and considering them for the open and closed models of RTS.

\begin{tabular}{|c|c|c|c|c|c|c|c|c|}
\hline$T$ & $\begin{array}{c}E_{13}^{\mathrm{c}} / \\
\tilde{E}_{13}^{\mathrm{c}}\end{array}$ & $\begin{array}{c}E_{13}^{o} / \\
\tilde{E}_{13}^{o} \\
\end{array}$ & $E_{13}^{\exp }$ & \begin{tabular}{|c|}
$\Gamma_{13}^{\mathrm{c}} /$ \\
$\tilde{\Gamma}_{13}^{\mathrm{c}}$ \\
\end{tabular} & $\begin{array}{c}\Gamma_{13}^{\mathrm{o}} / \\
\tilde{\Gamma}_{13}^{\mathrm{o}} \\
\end{array}$ & $\Gamma_{13}^{\exp }$ & \begin{tabular}{|c|}
$E_{12}^{\mathrm{c}} /$ \\
$\tilde{E}_{12}^{\mathrm{c}}$
\end{tabular} & $\begin{array}{c}E_{12}^{\mathrm{o}} / \\
\tilde{E}_{12}^{\mathrm{o}}\end{array}$ \\
\hline$[\mathrm{K}$ & \multicolumn{8}{|c|}{$[\mathrm{meV}]$} \\
\hline 10 & $\begin{array}{c}231.1 / \\
230.4\end{array}$ & $\begin{array}{c}240.9 / \\
240.2\end{array}$ & 233.1 & \begin{tabular}{|c|}
$0 /$ \\
0.18 \\
\end{tabular} & \begin{tabular}{|c|}
$8.7 /$ \\
8.9
\end{tabular} & 0.0 & \begin{tabular}{|c|}
$31.9 /$ \\
31.4
\end{tabular} & $\begin{array}{r}32.9 / \\
32.4\end{array}$ \\
\hline 00 & $\begin{array}{r}220.0 / \\
218.0\end{array}$ & $\begin{array}{c}229.6 / \\
227.6\end{array}$ & 227.1 & $\begin{array}{c}0 / \\
2.01\end{array}$ & $\begin{array}{l}9.9 / \\
13.4\end{array}$ & 16.4 & $\begin{array}{r}30.9 / \\
30.4\end{array}$ & $\begin{array}{c}32.1 / \\
31.6\end{array}$ \\
\hline
\end{tabular}

Using the obtained results for spectral parameters and renormalized due to electron-phonon interaction ones, we calculated the energies and widths of absorption bands for quantum transition $1 \rightarrow 3$ and differences between the energies of second and first state $\left(E_{13}^{(\mathrm{o}, \mathrm{c})}=\right.$ $\left.E_{3}^{(\mathrm{o}, \mathrm{c})}-E_{1}^{(\mathrm{o}, \mathrm{c})}, \Gamma_{13}=\Gamma_{1}+\Gamma_{1}, E_{12}^{(\mathrm{o}, \mathrm{c})}=E_{2}^{(\mathrm{o}, \mathrm{c})}-E_{1}^{(\mathrm{o}, \mathrm{c})}\right)$ without taking into account phonons and considering them $\left(\tilde{E}_{13}^{(\mathrm{o}, \mathrm{c})}=E_{13}^{(\mathrm{o}, \mathrm{c})}+\Delta_{3}-\Delta_{1}, \tilde{\Gamma}_{13}=\Gamma_{13}+\gamma_{3}+\gamma_{1}\right.$, $\tilde{E}_{12}^{(\mathrm{o}, \mathrm{c})}=E_{12}^{(\mathrm{o}, \mathrm{c})}+\Delta_{2}-\Delta_{1}$ ) for the open (o) and closed (c) models of three-well RTS. These magnitudes, together with the respective ones obtained in experimental paper [3] are presented in Table III at cryogenic and room temperatures. It is clear that the energies of absorption band at $T=10 \mathrm{~K}$ and $T=300 \mathrm{~K}$ in both models are similar. However, the results of open model (with electronphonon interaction) somewhat better coincide with experimental data. On the contrary to the closed model, where the width of absorption band arising at quantum transition between the stationary states is caused only by interaction with phonons, in open one it is also caused by the decays due to the finite life times of quasi-stationary states. Consequently the widths of absorption band obtained in closed model differ from the experimental data, while that of the open one correlate with them well. According to Ref. [3], the width of the band increases and its position shifts into the low-energy region at the increasing temperature. Herein, Tables II and III prove that the temperature broadening and short-wave shift of absorption band due to the weak electron-phonon interaction are small comparing to its broadening due to the decreasing life times of electron quasi-stationary states and long-wave shift caused by the decreasing heights of potential barriers because of smaller differences between the widths of energy gaps for the wells and barriers at bigger temperature.

\section{Conclusions}

Using the model of effective mass and rectangular potential for the electrons and dielectric continuum one for the optical I- and L-phonons, we present the theoretical description of electron-phonon interaction in closed three-well nanostructure $\operatorname{In}_{0.53} \mathrm{Ga}_{0.47} \mathrm{As} / \mathrm{In}_{0.52} \mathrm{Al}_{0.48} \mathrm{As}$.
The Hamiltonian of the system is obtained in the representation of second quantization over all variables. The renormalized parameters of electron spectrum (energy shift and decay rate) are calculated within the method of the Green functions at cryogenic and room temperatures. The contributions of different mechanisms of electron-phonon interaction into the renormalized electron states are studied as functions of three-well RTS configuration at the example of expanded active region of cascade of experimental QCD [3]. It is shown that at all conditions, the contribution produced by I-phonons is several times bigger than that of L-phonons.

Using the solution of complete Schrödinger equation, we studied the spectral parameters (resonance energies and widths) of electron quasi-stationary states and active conductivity caused by quantum transitions due to interaction of electrons with electromagnetic field in threewell RTS. It is established that phonons, at any temperature, weakly affect on the electron energy due to the weak electron-phonon binding. Thus, their contribution into the formation of electromagnetic field absorption band, arising due to the quantum transition, is small.

The long-wave shift and broadening of absorption band at higher temperature is, mainly, caused by the decreasing heights of RTS potential barriers due to the different magnitude of energy gaps for the wells and barriers, depending on temperature. The theoretical results well coincide with the experimental data [3].

\section{References}

[1] J. Faist, F. Capasso, D.L. Sivco, C. Sirtori, A.L. Hutchinson, A.Y. Cho, Science 264, 533 (1994).

[2] C. Gmachl, F. Capasso, D.L. Sivco, A.Y. Cho, Rep. Prog. Phys. 64, 1533 (2001).

[3] D. Hofstetter, M. Graf, T. Aellen, J. Faist, L. Hvozdara, S. Blaser, Appl. Phys. Lett. 89, 061119 (2006).

[4] M. Graf, N. Hoyler, M. Giovannini, J. Faist, D. Hofstetter, Appl. Phys. Lett. 88, 241118 (2006).

[5] S. Kumar, Q. Hu, J.L. Reno, Appl. Phys. Lett. 94, 131105 (2009).

[6] A. Bismuto, M. Beck, J. Faist, Appl. Phys. Lett. 98, 191104 (2011).

[7] L. Tombez, J. Di Francesco, S. Schilt, G. Di Domenico, J. Faist, P. Thomann, D. Hofstetter, Opt. Lett. 36, 3109 (2011).

[8] H. Schneider, H.C. Liu, S. Winnerl, O. Drachenko, M. Helm, J. Faist, Appl. Phys. Lett. 93, 101114 (2008).

[9] A. Buffaz, M. Carras, L. Doyennette, A. Nedelcu, X. Marcadet, V. Berger, Appl. Phys. Lett. 96, 172101 (2010).

[10] S. Sakr, E. Giraud, A. Dussaigne, M. Tchernycheva, N. Grandjean, F.H. Julien, Appl. Phys. Lett. 100, 181103 (2012).

[11] C. Jirauschek, T. Kubis, Appl. Phys. Rev. 1, 011307 (2014). 
[12] M. Lindskog, J.M. Wolf, V. Trinite, V. Liverini, J. Faist, G. Maisons, M. Carras, R. Aidam, R. Ostendorf, A. Wacker, Appl. Phys. Lett. 105, 103106 (2014).

[13] J. Faist, Quantum Cascade Lasers, Oxford University Press, Oxford 2013.

[14] R. Terazzi, J. Faist, New J. Phys. 12, 033045 (2010).

[15] F.R. Giorgetta, E. Baumann, M. Graf, Q. Yang, C. Manz, K. Kohler, H.E. Beere, D.A. Ritchie, E. Linfield, A.G. Davies, Y. Fedoryshyn, H. Jackel, M. Fischer, J. Faist, D. Hofstetter, J. Quantum Electron. 45, 1039 (2009).

[16] R. Betancourt-Riera, R. Rosas, I. Marín-Enriquez, R. Riera, J.L. Marín, J. Phys. Condens. Matter 17, 4451 (2005).

[17] N.V. Tkach, Yu.A. Seti, Low Temp. Phys. 35, 556 (2009).

[18] M.V. Tkach, Ju.O. Seti, O.M. Voitsekhivska, Condens. Matter Phys. 14, 43702 (2011).

[19] M.V. Tkach, Ju.O. Seti, O.M. Voitsekhivska, Acta Phys. Pol. A 124, 94 (2013).

[20] M.V. Tkach, Ju.O. Seti, I.V. Boyko, O.M. Voitsekhivska, Rom. Rep. Phys. 65, 1443 (2013).

[21] E. Saczuk, J.Z. Kaminski, Phys. Status Solidi B 240, 603 (2003).

[22] F.H.M. Faisal, J.Z. Kaminski, E. Saczuk, Phys. Rev. A 72, 023412 (2005).
[23] N. Mori, T. Ando, Phys. Rev. B 40, 6175 (1989).

[24] J.-J. Shi, B.C. Sanders, S.-H. Pan, Eur. Phys. J. B 4, 113 (1998).

[25] Z.W. Yan, X.X. Liang, Int. J. Mod. Phys. B 15, 3539 (2001)

[26] Z.W. Yan, S.L. Ban, X.X. Liang, Int. J. Mod. Phys. B 17, 6085 (2003).

[27] B.H. Wu, J.C. Cao, G.Q. Xia, H.C. Liu, Eur. Phys. J. B 33, 9 (2003).

[28] J.G. Zhu, S.L. Ban, Eur. Phys. J. B 85, 140 (2012)

[29] M.A. Stroscio, M. Dutta, Phonons in Nanostructures, Cambridge University Press, Cambridge 2001.

[30] L.D. Landau, E.M. Lifshitz, Quantum Mechanics: Non-Relativistic Theory, Vol. 3, 3rd ed., Pergamon Press, London 1977.

[31] D.F. Nelson, R.C. Miller, D.A. Kleinman, Phys. Rev. B 35, 7770(R) (1987)

[32] A.A. Abrikosov, L.P. Gorkov, I.E. Dzyaloshinski, Methods of Quantum Field Theory in Statistical Physics, Prentice Hall, Englewood Cliffs 1963.

[33] G.H. Davies, The Physics of Low-Dimensional Semiconductor, Cambridge University Press, Cambridge 1998. 